Research Article

\title{
Cloud Computing and Internet of Things in the Evaluation of Ecological Environment Quality in Rural Tourist Areas in Smart Cities
}

\author{
Ling Ding and Yezhang Liang $\mathbb{D}$ \\ Guangxi Research Institute of Culture and Tourism, Guilin Institute of Tourism, Guilin 541004, Guangxi, China \\ Correspondence should be addressed to Yezhang Liang; lyz@gltu.edu.cn
}

Received 23 June 2021; Revised 17 July 2021; Accepted 16 August 2021; Published 29 August 2021

Academic Editor: Sang-Bing Tsai

Copyright (C) 2021 Ling Ding and Yezhang Liang. This is an open access article distributed under the Creative Commons Attribution License, which permits unrestricted use, distribution, and reproduction in any medium, provided the original work is properly cited.

\begin{abstract}
With the increase of tourist environment, the real-time monitoring of ecological environment has become a concern. This study mainly discusses the application of cloud computing and Internet of things in the evaluation of ecological environment quality of rural tourism areas in a smart city. In this study, the real-time monitoring of the atmosphere, water, and meteorological data is collected through the GPRS data transmission module and then sent back to the local server by the GPRS network, and the obtained non-real-time and real-time data are used to establish the ecological monitoring database, the database analysis of its information, and get real-time data, monthly data, and longer cycle data. In the cloud GIS platform, there are multiple subnodes. The split tasks can be processed by each subnode through a map, and the results after processing can be summarized through reduce, which completes the implementation process of the whole idea of map reduce. Monitoring station management is mainly to establish monitoring stations in rural tourism areas and collect first-hand environmental monitoring data by using temperature, humidity, infrared, ultrasonic, and other sensors and cameras. The monitoring objects are the air quality, water quality, meteorology, etc. of the scenic area, mainly showing the location of monitoring stations and the placement of sensors. At the same time, an LED screen is set at the monitoring station to display the air quality data of the scenic spot. The data content is introduced into the DPSIR model, combined with social and economic data; according to the ecological health grading evaluation standard, the evaluation score and health grade are obtained and the ecological health status of rural tourism area is judged and evaluated. When the amount of data is less than $500 \mathrm{MB}$, there is little difference between the storage speed of the cloud GIS platform and single machine, but with the continuous increase of the amount of data, the storage speed of the cloud GIS platform is significantly higher than that of a single machine. This study is helpful in improving the ecological environment quality of rural tourism areas.
\end{abstract}

\section{Introduction}

Foreign research on ecotourism and original ecotourism mainly focus on how to achieve the purpose of natural area protection in the process of ecotourism development and the role of relevant factors and stakeholders in achieving the common goal; domestic research is mainly focused on the theory of the development planning and management of the original ecotourism resources, and there are also some results about the development mode of the original ecotourism and the protection of ecological vulnerability.
The Internet of things and cloud GIS is a spatial database based on the unified geographical coordinates, which uses the geoscience model to analyze the spatial data, manage and analyze the environment, resources, and other spatial information, and provide a variety of spatial and dynamic geographic information in real time. The GIS spatial analysis system selectively collects and analyzes various kinds of information, provides tourism resources spatial association data for tourism development decision makers, and provides support for tourism management decision making. This is also one of the main purposes and tasks of establishing a spatial analysis system based on cloud GIS. 
Mobile edge cloud computing is a new paradigm. Chen $\mathrm{W}$ believes that mobile edge cloud computing (MECC) has become an attractive solution for enhancing the computing and storage capacity of mobile devices (MDs) by leveraging the available resources at the edge of the network. He first formulated the multiuser and multitask computing offloading problem for the green MECC and then used the Lyapunov optimization method to determine the energy harvesting strategy how much energy each WD needs to collect and the task offloading schedule: a set of computing to be accepted into the mobile edge cloud unloading request, assigned to each WD group that has allowed the unloading request, and how much workload is to be processed in the assigned WD. His research put forward the concept of MD, but did not briefly describe its performance [1]. Cloud computing allows users to consume various IT resources on demand and has low management overhead. Han et al. believes that customers may face new security risks when using cloud computing platforms. He chose to solve the problem from different angles, by studying how to improve the virtual machine allocation strategy, so that it is difficult for the attacker to colocate with the target. Specifically, he defined security indicators used to assess attacks, modeled these indicators, and compared the difficulty of achieving coexistence under three common policies and designed a new strategy that not only mitigates the threat of attacks but also meets the needs of work quantity balance and low power consumption requirements. His research scheme is not novel enough [2]. Chen et al. formulated the decisionmaking problem of distributed computing offloading among mobile device users. He analyzed the structural nature of the game and proved that the game has limited improvements. Then, he designed a distributed computing shunt algorithm. The game theory method he adopted is unreasonable [3]. Xia et al. researched the performance improvement of the centralized cloud and integrated optical fiber wireless (FiWi) access network supporting MEC. He proposed a novel unified resource management solution that combines centralized cloud computing and MEC computing offloading activities into the basic FiWi dynamic bandwidth allocation process. By using time division multiple access, both MEC and cloud traffic are arranged outside the transmission time slot of FiWi traffic. He developed an analysis framework for the data packet delay and response time efficiency of cloud and broadband access traffic. The integrated optical fiber wireless he proposed has no specific performance test indicators and lacks logic [4].

This study mainly discusses the application of cloud computing and Internet of things in the evaluation of ecological environment quality of rural tourism areas in a smart city. In this study, the real-time monitoring of the atmosphere, water, and meteorological data is collected through the GPRS data transmission module and then sent back to the local server by the GPRS network, and the obtained non-real-time and real-time data were to establish the ecological monitoring database, the database analysis of its information, and get real-time data, monthly data, and longer cycle data. In the cloud GIS platform, there are multiple subnodes. The split tasks can be processed by each subnode through a map, and the results after processing can be summarized through reduce, which completes the implementation process of the whole idea of map reduce. Monitoring station management is mainly to establish monitoring stations in rural tourism areas and collect firsthand environmental monitoring data by using temperature, humidity, infrared, ultrasonic, and other sensors and cameras. The monitoring objects are the air quality, water quality, meteorology, etc. of the scenic area, mainly showing the location of monitoring stations and the placement of sensors.

\section{Ecological Environment in Rural Tourist Areas}

2.1. Rural Tourism. Rural tourism is not only an ancient way of tourism but also a trendy way of tourism for modern urbanites. Rural tourism is an inevitable product in the development of modern tourism $[5,6]$. The countryside was originally a conservative and relatively backward place with conservative economic, political, and cultural traditions, but now, it has become a situation that many urbanites yearn and pursue, which undoubtedly makes people puzzled. Modern society pursues nature and returns to the original. As the most primitive, most natural, most harmonious, most peaceful, and most charming place in society, the countryside is an ideal paradise for modern cities to escape the noise, relieve work pressure, and relax themselves. Rural tourism is popular nowadays. It has become one of the hotspots studied by scholars at home and abroad. With the continuous development of rural tourism, the main population of rural tourists has changed, and the source market is constantly being refined [7].

$$
\min F(x, z)=\sum_{k=1}^{m} \sum_{i=1}^{n} A_{k i} x_{k i} z_{i}+\sum_{i=1}^{n} \sum_{j=1}^{l} B_{i j} x_{i j} z_{i}+\sum_{k=1}^{m} \sum_{i=1}^{n} Z_{i} F .
$$

In the equation given above, $x$ and $z$ are the corresponding data management services $[8,9]$. The original tourism motives of rural tourists are expressed as the pursuit and enjoyment of the natural environment, return to nature, and enjoy the body and mind, and their environmental motives are the main tourism motives. However, as the development of rural tourism deepens, their tourism motives have also changed, showing diversity and uniqueness, not only pursuing the enjoyment of nature but also paying more attention to rural culture, experiential activities, and participatory activities outside the natural environment. The rural tourism market is also growing, the types of tourists are more diverse, and the needs are more diverse, which has also become an important content of practical and theoretical research [10]. For the overclocked carrier $f$, let the transmission phase be $\Phi$; then,

$$
\begin{aligned}
& G(t)=\left[\cos \left(2 \pi f_{0} t+\phi_{s}\right)+A\right] \cdot \cos \left(2 \pi f_{c} t+\phi_{c}\right), \\
& R(t)=\left[\cos \left(\pi f_{0} t+\phi_{r}\right)+A\right] \cdot \cos \left(\pi f_{c} t+\phi_{d}\right) . \\
& \text { Here, } \phi_{d}=\phi_{c}+f_{c} \cdot \tau_{d}[11,12] .
\end{aligned}
$$


2.2. Ecological Environment Quality. Ecological environmental quality evaluation not only provides a scientific quantitative basis for environmental management and decision making but also has important practical significance for regional sustainable development strategic decision making [13]. The objects of ecoenvironmental quality evaluation are mainly concentrated in countries, provinces, cities, and even specific areas. The evaluation methods used are also relatively diverse, mainly including the analytic hierarchy process, comprehensive index method, fuzzy evaluation method, principal component method, gray evaluation method, artificial neural network evaluation method, and other methods, and establish corresponding ecological environment evaluation according to the difference of evaluation objects system, such as the regional ecological environment quality evaluation system of provinces, cities, and ecologically fragile zones $[14,15]$.

Let $\Phi=\left(2 \pi n f_{0} / f_{s}\right)+\phi_{s}$ have $[16]$

$$
\begin{aligned}
& u=\left[\cos \left(\frac{2 \pi n f_{0}}{f_{s}}+\phi_{s}\right)+A\right] \cdot \cos \left(\frac{2 \pi n f_{c}}{f_{s}}\right), \\
& y=\left[\cos \left(\frac{2 \pi n f_{0}}{f_{s}}+\phi_{r}\right)+A\right] \cdot \cos \left(\frac{2 \pi n f_{c}}{f_{s}}+\phi_{d}\right) .
\end{aligned}
$$

Among them, $f_{s}$ is the sampling frequency [17]. Ecological quality evaluation is to select a certain evaluation index system and quality standard within a certain space and time range and use certain methods to evaluate the pros and cons of the ecological environment quality of a certain area and the influence relationship between various factors. The rural tourist area is a specific area, which is different from a large area such as a province or a country, but as an independent subsystem, its own system is complete, and there is also a certain relationship between factors. The method and theory of ecological environment quality evaluation should also be applicable [18, 19].

The choice of ecological environment quality evaluation indicators differs greatly due to the different research angles and research purposes. From the perspective of tourists, it focuses on the evaluation of ecological environmental factors that have a greater impact on tourists' sight, hearing, touch, and other senses. The United Nations integrated the advantages of the PSR model and the DSR model and proposed the DPSIR model for comprehensive analysis and description of environmental issues and their relationship with social development. The DPSIR concept is based on the extension of the PSR model and the DSR model. Social factors such as society and population act as drivers to promote or affect the ecological environment [20]. Average relative precipitation utilization efficiency, RUE, is

$$
R U E=\frac{\mathrm{RUE}_{1}-\mathrm{RUE}_{0}}{\mathrm{RUE}_{0}} .
$$

Maximum relative precipitation utilization efficiency:

$$
\mathrm{RUE}_{\text {max }}=\frac{\mathrm{RUE}_{\mathrm{ex}}-\mathrm{RUE}_{0}}{\mathrm{RUE}_{0}} .
$$

$\mathrm{RUE}_{0}$ is the minimum value of maximum precipitation utilization efficiency [21]. Organic matter content $H$ is an important indicator for calculating soil parameters:

$$
H=\frac{2.6}{B 1+B 2+B 3}-1.6 \text {. }
$$

Here, $B 1$ is the reflectivity of red light [22].

2.3. Cloud GIS. Compared with cloud computing, the key technologies of cloud GIS implementation are mainly reflected in a "spatial" feature. It needs to consider more fully the spatial problems on the basis of cloud computing implementation, such as distributed storage, access, and processing of spatial data, study how to combine this spatial feature of GIS with the coding mode of cloud computing, and realize the seamless link between the them [23]. According to the requirements of the optimal allocation of system resources, the following functions are implemented:

$$
\begin{aligned}
& k(t)=\sum_{i=1}^{n} p_{i} F_{i}, \\
& R_{i}=E^{-r t}\left[a_{1} p_{s}+a_{2} p_{E}+a_{3} p_{0}\right] .
\end{aligned}
$$

Among them, $p_{i}$ is the value of big data asset $F$ [24]. Let $G$ be the point set of the real 3D model and $R$ be the test point set of the model obtained by the reconstruction algorithm:

$$
T=\min _{g \in G}|\gamma-G| \text {. }
$$

The precision of the reconstructed point set $\gamma$ is defined as the cumulative result of all points [25].

$$
P(D)=\frac{100}{|R|} \sum_{r \in R}\left[e_{r \rightarrow G}<D\right] .
$$

For any real three-dimensional point, $g \in G$ :

$$
E=\min _{r \in R}|g-r| .
$$

The recall rate of the reconstructed model $R$ for a given distance threshold $d$ is defined as

$$
W(d)=\frac{1}{|G|} \sum_{g \in G} X .
$$

The construction of the cloud computing environment is the basis for the construction of the entire cloud GIS platform, including software and hardware environments such as network, storage, and virtualization. By using basic hardware facilities, virtualization software is used to build a server virtualization environment, and then, database software is deployed on virtual machines and GIS server software cluster environment [26]. The value of $k$ after iteration is recorded as $P$, and the prediction function is as follows:

$$
W(C, P)=\sqrt{W^{2}(C)+B^{2}(C)} .
$$

In terms of popularization and civilianization, private cloud will provide the public with more public information 
online services, such as transportation, catering, renting, and tourism. With the support of an efficient and stable architecture, cloud GIS will break the original "professional circle," bring more value-added services related to spatial information to the public, and bring users who wish to build their own GIS application platform to achieve a revolution in GIS itself. Sexual changes continue to expand the market and serve the masses $[27,28]$.

Arc GIS Online is an important part of the Arc GIS series products. It can be seamlessly integrated with other products in the series. Other series products can fully use various service resources in Arc GIS Online to complete their own operations. At the same time, they can use Arc GIS Online to interact with other products. The function of the product is expanded. In the cloud platform, users can not only use data but also the cloud platform provides computing power that is incomparable to ordinary stand-alone computers. Distributed computing and parallel computing are used to greatly increase the speed of data processing and accelerate the progress of scientific research.

\section{Evaluation Experiment of Ecological Environment Quality in a Rural Tourism Area}

3.1. Ecological Monitoring System in Rural Tourist Areas. This research collects the ecological environmental factors of rural tourist areas, including air quality, temperature, humidity, negative oxygen ion concentration, sulfur dioxide concentration in the tourist area, and weather information. We used various sensors such as temperature, humidity, infrared, ultrasound, and cameras to collect first-hand environmental monitoring data, design the ecological environment monitoring system of rural tourist areas, and transmit various indicator data back to the system for analysis and processing. Superimposed on dynamic monitoring data, each monitoring point can display the monitoring data of this place and the monitoring data of a period of time in the past, realizing all-weather, all-round $24 \mathrm{~h}$ realtime dynamic monitoring of multiple controlled areas. We provide visual management for relevant departments to take timely response strategies and control measures.

3.2. Overall System Design. The ecological environment monitoring system of the rural tourist area is shown in Figure 1. It obtains its own rural social and economic indicator data, as well as ecological environment (water quality, air quality, and meteorological) data, and automatically collects, processes, integrates, displays, and queries statistics; the quasi-real-time monitoring of the atmosphere, water, weather, and remote sensing images based on realtime monitoring is the original data; the real-time monitoring of the atmosphere, water, and weather data is collected through the GPRS data transmission module and then transmitted back to the local server through the GPRS network. An ecological monitoring database with real-time data is established. The database analyzes its information to obtain real-time data, monthly data, and longer-period data. According to the ecological health classification evaluation criteria, to obtain evaluation scores and health levels and judge and evaluate the ecological health status of rural tourism areas, in the cloud GIS platform, there are multiple subnodes. The split tasks can be handed over to each subnode for processing through a map, and the results after processing are aggregated through reduce, which will greatly improve efficiency. The execution process of the whole idea of map reduce is completed.

3.3. Cloud GIS Platform. Cloud computing mainly provides three service modes, namely, software and service, platformas-a-service, and infrastructure-as-a-service. GIS is put into the cloud to build the cloud GIS platform. The original interface, module, and other aspects need to be changed in order to connect with the existing cloud computing platform. Cloud GIS can be constructed in the following four ways: geographic information content as a service, geographic information software-as-a service, geographic information platform-as-a-service, and geographic information infrastructure-as-a-service.

3.4. Database Design. The purpose of the database design is to collect and integrate the complex information of rural tourist areas, store and efficiently manage the data of ecological environment factors in rural tourist areas, and establish an ecological monitoring database. Due to the large amount of monitoring data and fast update, it requires strong data access and interaction capabilities. The daily record of real-time monitoring data needs to be extracted and processed by the GPRS data transmission equipment to be displayed in the data management and display, and the ecological environment monitoring database is used to read the data. The ecological monitoring system database is designed with a process-oriented data update function, and the platform can automatically record and release the temporal information of the data according to the data update situation. Data analysis can read database data, generate statistical reports, display chart, etc. Among them, the database structure includes two types of tables: the first type is user management tables, back-end management log tables, etc.; the second type is monitoring data tables, which are mainly basic data, collector tables and equipment tables for non-real-time and real-time data, and the control instruction list.

3.5. Functional Module Design. The main functions of the rural ecological environment monitoring system include platform management, monitoring station management, equipment management, data management, data analysis, and data expression. The functional module design is as follows.

3.5.1. Platform Management. Platform management includes user, security, and platform management. It is mainly to create users, add and delete user name information such as passwords and names, and perform security management such as user management in the background of the platform, 
setting user permissions, setting administrator status, platform setting operation log records, internal and external network management, and other security management.

3.5.2. Monitoring Station Management. Monitoring station management is mainly to establish monitoring stations in rural tourist areas. The monitoring objects are the air quality, water quality, and weather of the scenic area and mainly display the location of the monitoring station and the placement of sensors. At the same time, LED screens are set up at the monitoring sites to display air quality data in the scenic area.

3.5.3. Equipment Management. Equipment management is the use of equipment management for each monitoring data. It is mainly to realize the functions of adding equipment, deleting equipment, viewing equipment configuration, uploading equipment configuration information, downloading equipment information, and modifying equipment parameters, mainly modifying equipment table, serial port channel, geographic location information, administrative district number and project, etc.

3.5.4. Data Management. The data management function is the three modules of data collection, data processing, and data backup. It uses the GPRS data transmission equipment to obtain data, performs data preprocessing, and uses software to write to the local server, establish an ecological monitoring database, and obtain real-time monitoring data from the local server. Unified and integrated management of various service data resources is used to ensure the consistency of data resources. The ecological monitoring system constructs monitoring data, attribute data, site data, and thematic data for seamless splicing and integrated management. At the same time, the data are backed up regularly. The platform can do a level-one incremental backup once a week and a full-database backup once a month.

3.5.5. Data Analysis. Data analysis includes three modules: query and retrieval, statistical analysis, and model analysis. Non-real-time socioeconomic index data, remote sensing data, and real-time monitoring data are queried and retrieved. Statistical analysis is to count monthly or longer cycle monitoring data, display in the form of charts, and use the data to predict the ecological status and pressure of rural tourism areas. Model analysis is to bring the social and economic data and annual monitoring data into the DPSIR model, calculate the evaluation results of the model, and determine the health status and health level of rural tourism areas.

3.5.6. Data Expression. Data expression includes thematic information display, comprehensive information display, and electronic map display. The data expression can satisfy all kinds of users' browsing and query of the monitoring data of rural tourist areas involving basic operating functions (roaming, zooming, ranging, etc.), statistical charts of social and economic data and real-time monitoring data in rural tourism areas, uploading and downloading remote sensing data in rural tourism areas, and monitoring the dynamic changes of the ecological environment in rural tourism areas, such as monitoring lakes' amount of water level change displayed on the air quality LED screen in rural scenic spots. The electronic map interactively displays the air quality, negative oxygen ion content, PM2.5 value, and meteorological information (including wind direction, humidity, and temperature) of each scenic spot. The air quality data are shown in Table 1.

\section{Results and Discussion}

According to the principle of selection of ecological environment quality evaluation indicators, the indicators are divided into three levels: target level $(A)$, support level $(B)$, and index level $(C)$. Among them, the target layer $(A)$ is the ecological environment quality $(A)$ and the support layer $(B)$ is divided into the natural environment $(B 1)$, the social environment (B2), and the human environment (B3). The ecological environmental indicators of rural tourist areas are shown in Table 2.

Understanding the characteristics of the market segmentation of rural tourism tourists is an important content of studying rural tourism ecological environment evaluation and the main basis for formulating rural tourism marketing strategies. We selected time points when the passenger flow is relatively large during the Spring Festival, Golden Week, weekends, etc. and conducted a random survey of local or out-of-town tourists visiting the scenic spot. The information of local or foreign tourists is shown in Table 3.

Regarding gender characteristics, the ratio of males to females in this survey is $1: 1.2$, with the majority of female tourists and fewer males; age characteristics: the frequency of selection for the 19-25 age group accounts for $24.4 \%$ of the sample, for the 26-40 age group, the segment is $36.3 \%$, and for the 41-60 age group, it is $19.4 \%$. The total selection frequency of the 19-60 age group accounts for $80.1 \%$ of the sample, indicating that the age group of tourists is mainly concentrated between 19 and 60 years of age. The specific comparison of each age group is shown in Figure 2.

The characteristics of education level: the proportion of junior high school to undergraduate level is $89.4 \%$. Most tourists have middle-school and high-school education, and there are fewer tourists with other education levels. The comparison of the proportions of different education levels is shown in Figure 3.

Household monthly income characteristics: income segments above 2501-8001 Yuan accounted for $99.3 \%$ of the sample, of which the 4001-6500 Yuan segment reached up to $34.3 \%$. The proportion of each household's monthly income segment is shown in Figure 4.

In the design of the questionnaire, "excellent $=5$ " and "extremely poor $=1$ " were used to evaluate the ecological environment quality. Therefore, the closer the mean is to " 5 ," the better the quality is, and the closer the mean is to " 1 ," the poorer the quality status is, the smaller the standard 


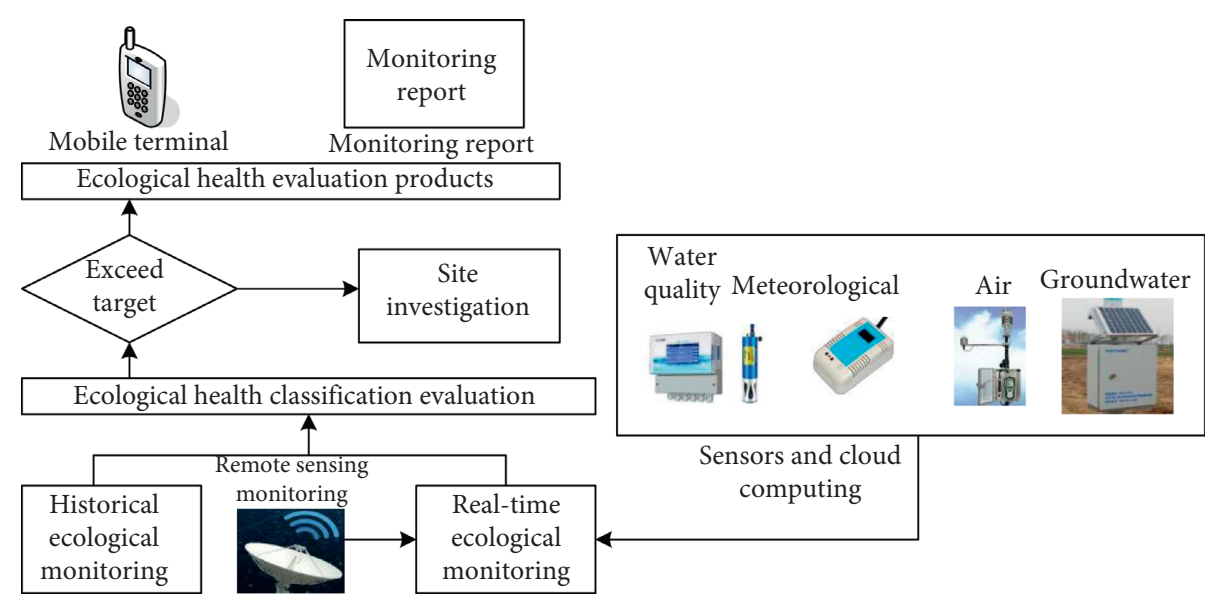

FIgURE 1: Ecological environment monitoring system in rural tourist areas.

TABLE 1: Air quality data.

\begin{tabular}{lcc}
\hline Substance & Numerical value & Unit \\
\hline $\mathrm{SO}_{2}$ & 10 & $\mathrm{mg} / \mathrm{m}^{3}$ \\
$\mathrm{NO}_{\mathrm{x}}$ & 50 & $\mathrm{mg} / \mathrm{m}^{3}$ \\
Particulates & 9 & $\mathrm{mg} / \mathrm{m}^{3}$ \\
Pressure & -20 & $\mathrm{~Pa}$ \\
$\mathrm{O}_{2}$ & 15 & $\%$ \\
Temperature & 37 & $\%$ \\
Flow & 9940 & ${ }^{\circ} \mathrm{C}$ \\
\hline
\end{tabular}

TABLE 2: Ecoenvironmental indicators of rural tourism areas.

Natural environment $(B 1)$

Ecological environment quality $(A)$
Social environment $(B 2)$

Humanistic environment (B3)
Water quality $(C 1)$

Air quality $(C 2)$

Climate comfort $(C 3)$

Resident warmth and friendliness (C6) (C3)

Location and traffic conditions (C7) (C3) Accommodation conditions (C8)

Local public security environment $(C 11)$

Local characteristic culture $(\mathrm{C} 12)$

Resource cultural connotation $(\mathrm{C} 13)$ deviation is, and the more consistent the evaluation of tourists is. The specific evaluation is shown in Table 4.

It can be seen from Table 4 that tourists have the best evaluation of the quality of the natural environment $(B 1)$, the quality of the social environment $(B 2)$ is good, and the quality of the human environment (B3) is not good. Judging by the standard deviation, the standard deviations of the three are relatively close, indicating that tourists' evaluation of the quality of these three aspects is relatively consistent. Therefore, it is determined that, in terms of the quality of the ecological environment in rural tourism areas, the natural environment is the best, the social environment is better, and the human environment is not good. The ranking of the quality of the three is shown in Figure 5.

The descriptive statistical analysis of the quality of 9 indicators of the ecological environment is shown in Table 5.

It can be seen from Table 5 that tourists have the best quality evaluations of air quality (C2) and climate comfort (C3); environmental noise $(C 4)$ and green area $(C 5)$ are evaluated by tourists; for water quality $(C 1)$, tourism reception infrastructure (C9), and other factors, the evaluation of tourists is average, of which the water quality $(C 1)$ and tourism reception infrastructure (C9) are less satisfactory; the residents' warmth and friendliness (C6) is not well evaluated by tourists. The four factors, traffic conditions $(C 7)$ and accommodation conditions (C8), are the worst evaluated by tourists. Among them, the evaluation of accommodation conditions is the worst. The ranking of the average quality scores of some indicators is shown in Figure 6.

The comprehensive evaluation of the ecological environment quality of rural tourist areas involves the weight of each indicator and the actual score of the indicator. Therefore, the total score of ecological environmental quality is set as 100 points, and the theoretical score of ecological environmental quality $(100)=$ the theoretical score of natural environment (36.5) + the theoretical score of social environment $(31.7)+$ the theoretical score of humanistic environment (31.8), theoretical score of the 
TABLE 3: Information for local or foreign tourists.

\begin{tabular}{lccc}
\hline Influencing factors & Basic tourist information & Frequency & Proportion (\%) \\
\hline \multirow{2}{*}{ Gender } & Male & 88 & 55 \\
& Female & 72 & 45 \\
\hline \multirow{4}{*}{ Age } & Less than or equal to 18 & 22 & 13.8 \\
& $19-25$ & 39 & 24.4 \\
& $26-40$ & 58 & 36.2 \\
Education level & $41-60$ & 10 & 19.4 \\
& 61 or more & 10 & 6.2 \\
\hline
\end{tabular}

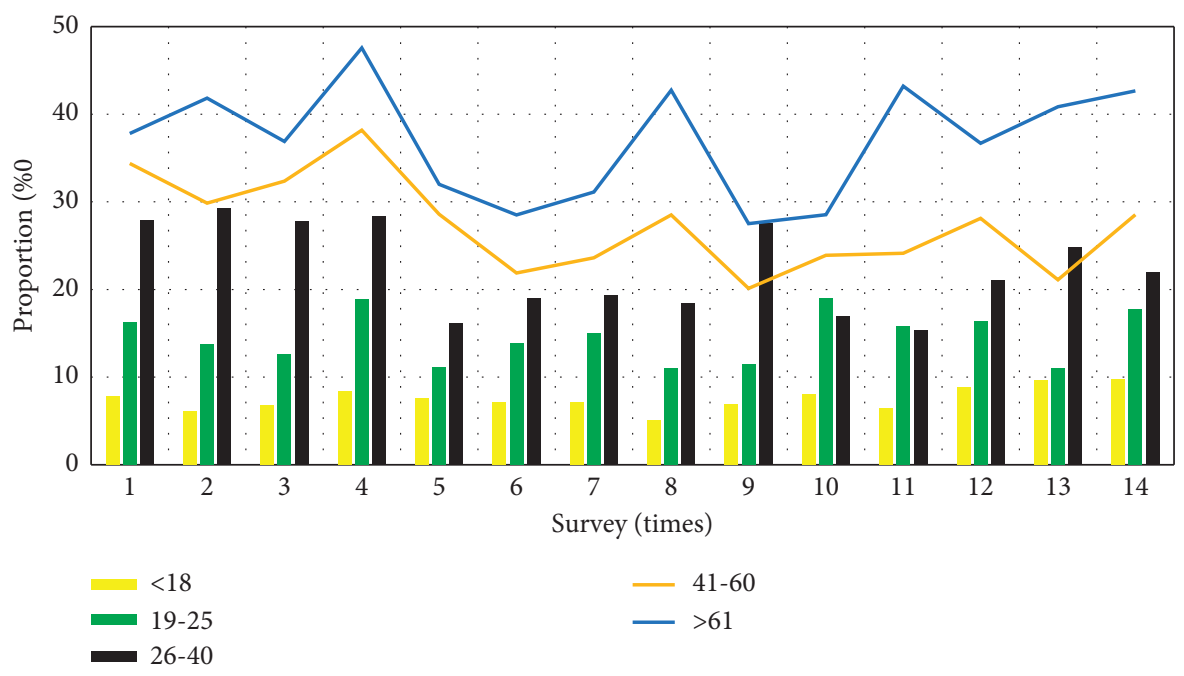

Figure 2: Comparison of the specific share of each age group.

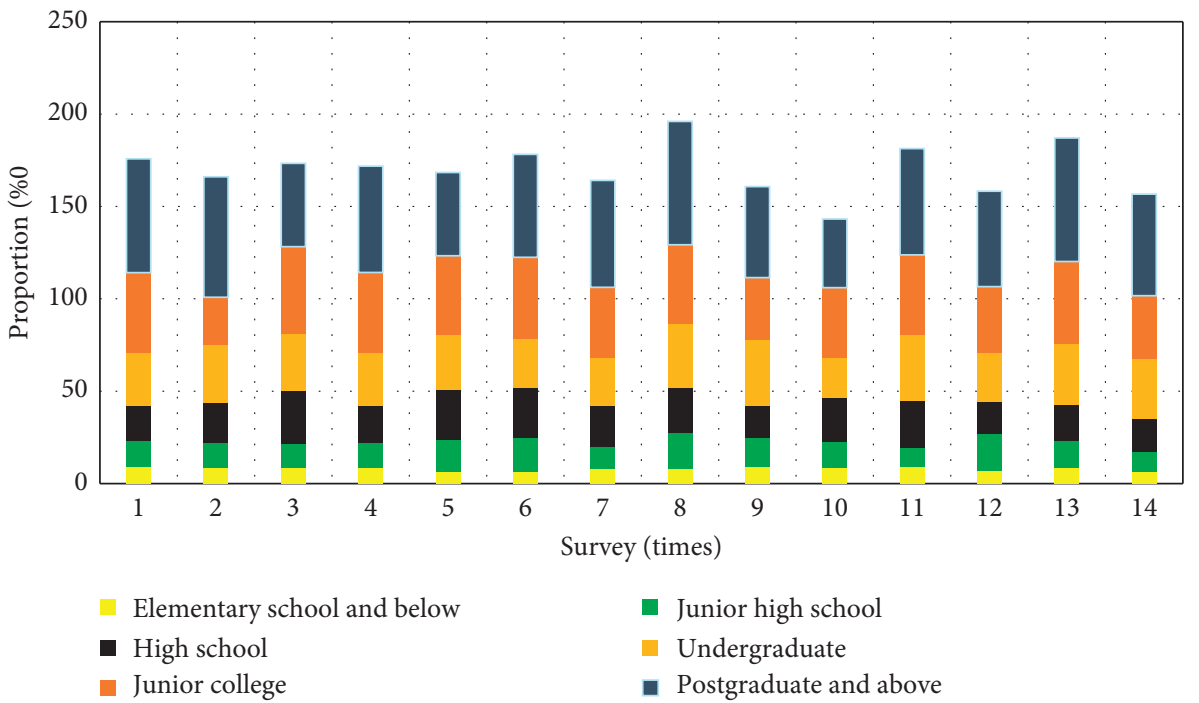

Figure 3: Comparison of the share of each degree of education.

indicator $=$ weight $*$ total score, the weight of the indicator is determined by the important average value of each indicator, and the theoretical score and weight of each indicator can be calculated by analogy. The comprehensive evaluation of ecological environment quality is shown in Table 6.

The importance-performance score of the index-level index is shown in Table 7 . 


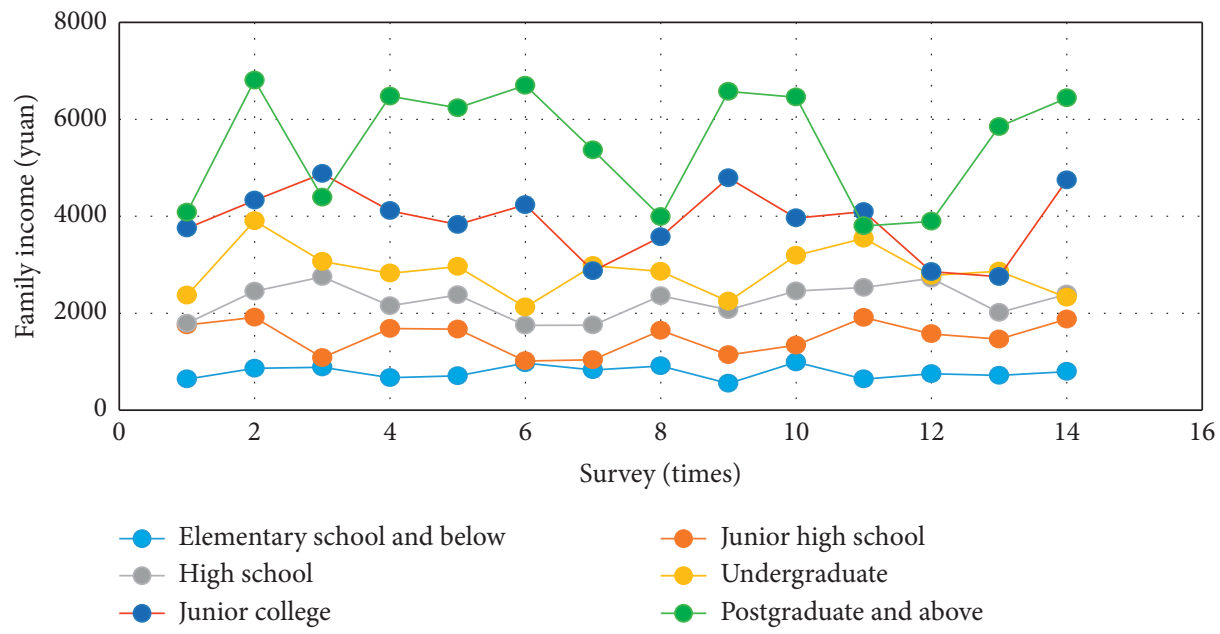

Figure 4: Proportion of each household's monthly income segment.

TABLE 4: Specific evaluation.

\begin{tabular}{lcccc}
\hline Index & Minimum & Max & Average value & Standard deviation \\
\hline Natural environment $(B 1)$ & 2 & 5 & 3.61 & 0.67 \\
Social environment $(B 2)$ & 2 & 5 & 3.44 & 0.63 \\
Humanistic environment $(B 3)$ & 1 & 5 & 3.40 & 0.60 \\
\hline
\end{tabular}

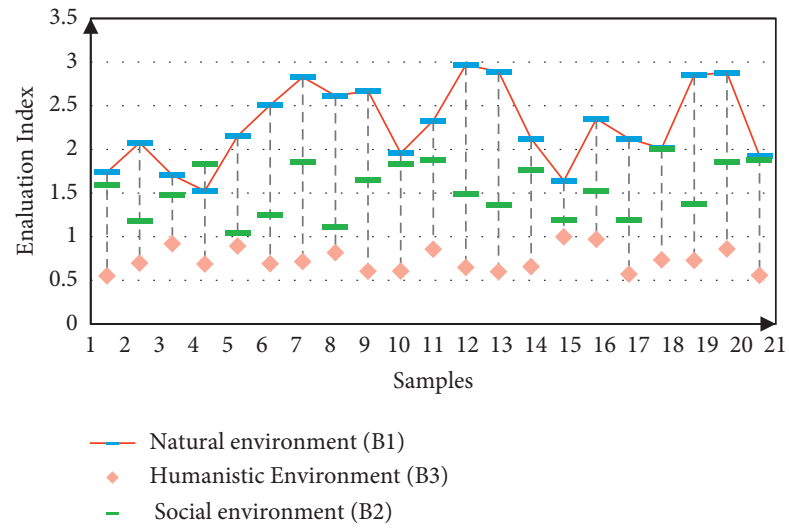

FIGURE 5: The ranking of the quality of the three.

TABLE 5: Descriptive statistics on the quality of 9 indicators of the ecological environment.

\begin{tabular}{lcc}
\hline Index & Average value & Standard deviation \\
\hline Water quality $(C 1)$ & 3.47 & 0.64 \\
Air quality $(C 2)$ & 3.68 & 0.63 \\
Climate comfort $(C 3)$ & 3.81 & 0.60 \\
Environmental noise $(C 4)$ & 3.58 & 0.61 \\
Green area $(C 5)$ & 3.56 & 0.62 \\
Resident warmth and friendliness $(C 6)$ & 3.33 & 0.62 \\
Location and traffic conditions $(C 7)$ & 3.28 & 0.64 \\
Accommodation conditions $(C 8)$ & 3.23 & 0.62 \\
Tourist reception infrastructure $(C 9)$ & 3.42 & 0.69 \\
\hline
\end{tabular}

The performance of the cloud GIS platform is shown in Figure 7. Regardless of whether it is based on a stand-alone or cloud GIS platform, the amount of data stored in the database is always linearly correlated with the storage time; that is, with the increase or decrease of the amount of stored data, the storage time will increase or decrease accordingly: 


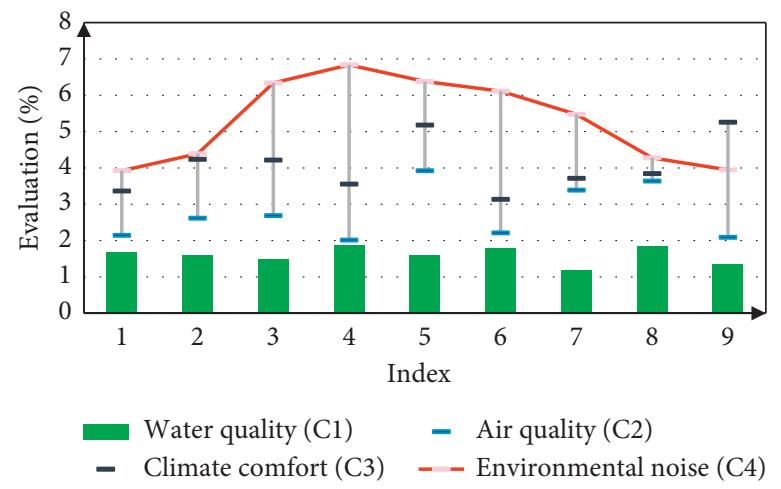

FIgURE 6: The ranking of the average size of the quality scores of some indicators.

TABLE 6: Comprehensive evaluation of ecological environment quality.

\begin{tabular}{lccc}
\hline Index & Mean importance & Weights & Index \\
\hline Natural environment $(B 1) 36.5$ & 4.7 & 0.365 & $\begin{array}{c}\text { Water quality }(C 1) \\
\text { Air quality }(C 2) \\
\text { Climate comfort }(C 3)\end{array}$ \\
\hline Social environment (B2) 31.7 & 4.1 & 0.317 & $\begin{array}{c}\text { Resident warmth and friendliness }(C 6) \\
\text { Location and traffic conditions }(C 7) \\
\text { Accommodation conditions }(C 8)\end{array}$ \\
\hline Humanistic environment (B3) 31.8 & 4.2 & 0.318 & $\begin{array}{c}\text { Local public security environment }(C 11) \\
\text { Resource cultural connotation }(C 12)\end{array}$ \\
\hline
\end{tabular}

TABLE 7: Importance of indicators at the index-level performance score.

\begin{tabular}{lcc}
\hline Factor & Importance average & Performance average \\
\hline Water quality $(C 1)$ & 4.68 & 3.47 \\
Air quality $(C 2)$ & 4.71 & 3.68 \\
Climate comfort $(C 3)$ & 4.25 & 3.81 \\
Environmental noise $(C 4)$ & 4.16 & 3.58 \\
Green area $(C 5)$ & 4.34 & 3.56 \\
Resident warmth and friendliness (C6) & 3.99 & 3.33 \\
\hline
\end{tabular}

when the amount of data is small (100 MB Below), the storage time of the cloud GIS platform and the stand-alone machine is not much different, and the storage time of the stand-alone machine is even shorter. However, as the amount of data increases, the time spent on a single machine is significantly longer than that of the cloud GIS platform, and this advantage of the cloud GIS platform continues to deepen. By analyzing the comparison chart of image storage speed $(\mathrm{MB} / \mathrm{S})$ based on a single machine and the cloud GIS platform, it can be found that there is a linear positive correlation between the storage data volume and storage speed based on cloud GIS platform; that is, as the amount of data increases, storage speed is also increasing. On the other hand, there is a positive correlation and then a negative correlation between the data volume and the speed of the storage based on a single machine. The inflection point occurs when the data volume is $300 \mathrm{MB}$; when the data volume is less than $500 \mathrm{MB}$, the cloud GIS platform and the storage speed of a single machine are not much different, and even the storage speed of a single machine was higher than that of the cloud GIS platform. However, as the amount of data continues to increase, the storage speed of the cloud GIS platform is significantly higher than the speed of a single machine, and this advantage continues to expand. 

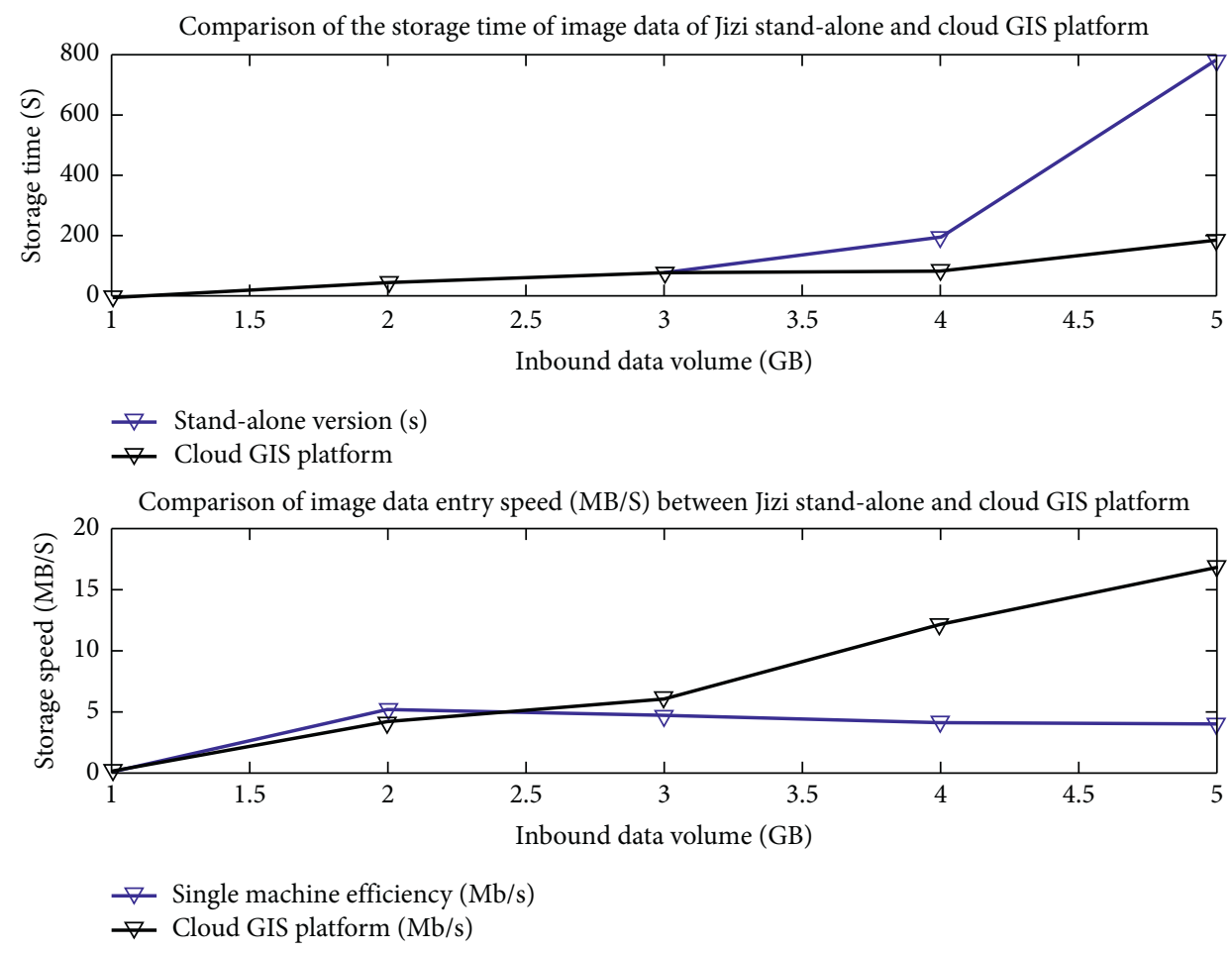

FIGURE 7: Cloud GIS platform performance.

\section{Conclusions}

The development of tourism is inseparable from the environment, so we must pay attention to the quality of tourism environment closely related to tourism. Only by establishing a good relationship with the tourism environment can the tourism industry walk out of a harmonious and low energy consumption development mode. This study mainly discusses the application of cloud computing and Internet of things technology in the evaluation of ecological environment quality of rural tourism areas in a smart city. In this study, the real-time monitoring of the atmosphere, water, and meteorological data is collected through the GPRS data transmission module and then sent back to the local server by the GPRS network; the obtained non-real-time and realtime data are used to establish the ecological monitoring database, the database analysis of its information, and get real-time data, monthly data, and longer cycle data. In the cloud GIS platform, there are multiple subnodes. The split tasks can be processed by each subnode through a map, and the results after processing can be summarized through reduce, which completes the implementation process of the whole idea of map reduce. In the future work, we should include the working environment into the environmental assessment standards, apply $3 \mathrm{~S}$ technology to the environmental monitoring of scenic spots, reasonably plan the ecotourism, and ensure the protection of the tourism environment.

\section{Data Availability}

No data were used to support this study.

\section{Conflicts of Interest}

The authors declare no conflicts of interest.

\section{References}

[1] W. Chen, D. Wang, and K. Li, "Multi-user multi-task computation offloading in green mobile edge cloud computing," IEEE Transactions on Services Computing, vol. 12, no. 5, pp. 726-738, 2019.

[2] Y. Han, J. Chan, T. Alpcan et al., "Using virtual machine allocation policies to defend against co-resident attacks in cloud computing," IEEE Transactions on Dependable \& Secure Computing, vol. 14, no. 1, pp. 95-108, 2017.

[3] X. Chen, L. Jiao, W. Li, and X. Fu, "Efficient multi-user computation offloading for mobile-edge cloud computing," IEEE/ACM Transactions on Networking, vol. 24, no. 5, pp. 2795-2808, 2016.

[4] Z. Xia, X. Wang, L. Zhang et al., "A privacy-preserving and copy-deterrence content-based image retrieval scheme in cloud computing," IEEE Transactions on Information Forensics \& Security, vol. 11, no. 11, pp. 2594-2608, 2016.

[5] B. P. Rimal, D. Pham Van, and M. Maier, "Mobile-edge computing versus centralized cloud computing over a converged FiWi access network," IEEE Transactions on Network and Service Management, vol. 14, no. 3, pp. 498-513, 2017.

[6] Q. Yan, F. R. Yu, Q. Gong, and J. Li, "Software-defined networking (SDN) and distributed denial of service (DDoS) attacks in cloud computing environments: a survey, some research issues, and challenges," IEEE Communications Surveys \& Tutorials, vol. 18, no. 1, pp. 602-622, 2016.

[7] R. Deng, R. Lu, C. Lai et al., "Optimal workload allocation in fog-cloud computing toward balanced delay and power consumption," IEEE Internet of Things Journal, vol. 3, no. 6, pp. 1171-1181, 2016. 
[8] M. Masdari, S. Valikardan, Z. Shahi, and S. I. Azar, "Towards workflow scheduling in cloud computing: a comprehensive analysis," Journal of Network and Computer Applications, vol. 66, pp. 64-82, 2016.

[9] Z. Cao, J. Lin, C. Wan et al., "Optimal cloud computing resource allocation for demand side management," IEEE Transactions on Smart Grid, vol. 8, no. 4, pp. 1943-1955, 2017.

[10] H. Cai, B. Xu, L. Jiang, and A. V. Vasilakos, "IoT-based big data storage systems in cloud computing: perspectives and challenges," IEEE Internet of Things Journal, vol. 4, no. 1, pp. 75-87, 2017.

[11] A. A. Soofi and M. Irfan Khan, "A review on data security in cloud computing," International Journal of Computer Applications, vol. 96, no. 2, pp. 95-96, 2017.

[12] H. Abbas, O. Maennel, and S. Assar, "Security and privacy issues in cloud computing," Annals of Telecommunications, vol. 72, no. 5-6, pp. 233-235, 2017.

[13] J. Shen, T. Zhou, X. Chen, J. Li, and W. Susilo, "Anonymous and traceable group data sharing in cloud computing," IEEE Transactions on Information Forensics and Security, vol. 13, no. 4, pp. 912-925, 2018.

[14] H. M. Sabi, F.-M. E. Uzoka, K. Langmia, and F. N. Njeh, "Conceptualizing a model for adoption of cloud computing in education," International Journal of Information Management, vol. 36, no. 2, pp. 183-191, 2016.

[15] S. Wang, K. Liang, J. K. Liu et al., "Attribute-based data sharing scheme revisited in cloud computing," IEEE Transactions on Information Forensics \& Security, vol. 11, no. 8, pp. 1661-1673, 2016.

[16] M. Guzek, P. Bouvry, and E. G. Talbi, "A survey of evolutionary computation for resource management of processing in cloud computing," IEEE Computational Intelligence Magazine, vol. 10, no. 2, pp. 53-67, 2015.

[17] M. Anshari, Y. Alas, and L. S. Guan, "Developing online learning resources: big data, social networks, and cloud computing to support pervasive knowledge," Education and Information Technologies, vol. 21, no. 6, pp. 1663-1677, 2016.

[18] S. Singh and I. Chana, "A survey on resource scheduling in cloud computing: issues and challenges," Journal of Grid Computing, vol. 14, no. 2, pp. 217-264, 2016.

[19] A. F. Barsoum and M. A. Hasan, "Provable multicopy dynamic data possession in cloud computing systems," IEEE Transactions on Information Forensics \& Security, vol. 10, no. 3, pp. 485-497, 2015.

[20] J. L. Tsai and N. W. Lo, "A privacy-aware authentication scheme for distributed mobile cloud computing services," IEEE Systems Journal, vol. 9, no. 3, pp. 805-815, 2015.

[21] M. Abdullahi, M. A. Ngadi, and S. I. M. Abdulhamid, "Symbiotic organism search optimization based task scheduling in cloud computing environment," Future Generation Computer Systems, vol. 56, pp. 640-650, 2016.

[22] E. Cavalcante, J. Pereira, M. P. Alves et al., "On the interplay of internet of things and cloud computing: a systematic mapping study," Computer Communications, vol. 89-90, pp. 17-33, 2016.

[23] A. B. Ali, T. Ali, and A. K. Wafaa, "Financial incentives for adopting cloud computing in higher educational institutions," Asian Social Science, vol. 13, no. 4, pp. 162-174, 2017.

[24] W.-L. Shiau and P. Y. K. Chau, "Understanding behavioral intention to use a cloud computing classroom: a multiple model comparison approach," Information \& Management, vol. 53, no. 3, pp. 355-365, 2016.

[25] A. Esfahani, G. Mantas, R. Matischek et al., "A lightweight Authentication mechanism for M2M communications in industrial IoT environment," IEEE Internet of Things Journal, vol. 6, no. 1, pp. 288-296, 2019.

[26] Q. Ye, W. Zhuang, X. Li, and J. Rao, "End-to-End delay modeling for embedded VNF chains in 5G core networks," IEEE Internet of Things Journal, vol. 6, no. 1, pp. 692-704, 2019.

[27] V. J. Magalhaes, A. M. Alberti, G. D. Scarpioni et al., “Advancing novagenesis architecture towards future internet of things," IEEE Internet of Things Journal, vol. 6, no. 1, pp. 215-229, 2019.

[28] S. Wang, J. Zhou, W. Xie et al., "An efficient file hierarchy attribute-based encryption scheme in cloud computing," IEEE Transactions on Information Forensics and Security, vol. 11, no. 6, pp. 1265-1277, 2016. 